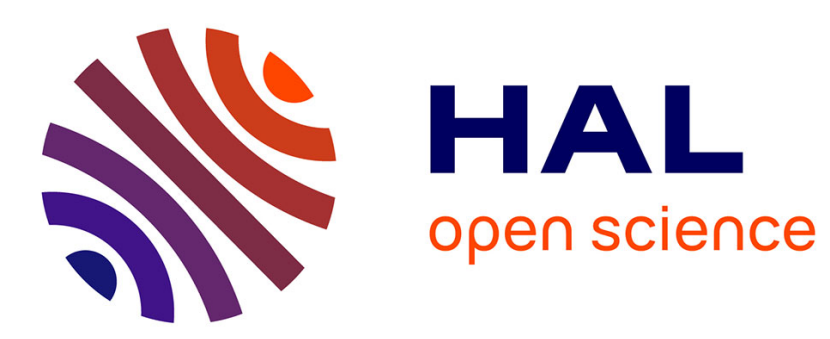

\title{
L'exercice sportif : entre douleur et plaisir
}

Stéphane Héas, Omar Zanna

\section{- To cite this version:}

Stéphane Héas, Omar Zanna. L'exercice sportif : entre douleur et plaisir. Revue des Sciences sociales, 2015, 53, pp.110-116. 10.4000/revss.2858 . hal-02172165

\section{HAL Id: hal-02172165 \\ https://hal.univ-rennes2.fr/hal-02172165}

Submitted on 3 Jul 2019

HAL is a multi-disciplinary open access archive for the deposit and dissemination of scientific research documents, whether they are published or not. The documents may come from teaching and research institutions in France or abroad, or from public or private research centers.
L'archive ouverte pluridisciplinaire HAL, est destinée au dépôt et à la diffusion de documents scientifiques de niveau recherche, publiés ou non, émanant des établissements d'enseignement et de recherche français ou étrangers, des laboratoires publics ou privés. 


\section{L'exercice sportif : entre douleur et plaisir}

Athletic exercise between pain and pleasure

\section{Stéphane Héas et Omar Zanna}

\section{(2) OpenEdition}

\section{Journals}

Édition électronique

URL : http://journals.openedition.org/revss/2858

DOI : $10.4000 /$ revss.2858

ISSN : 2107-0385

\section{Éditeur}

Presses universitaires de Strasbourg

\section{Édition imprimée}

Date de publication : 22 septembre 2015

Pagination : 110-116

ISBN : 978-2-86820-918-4

ISSN : 1623-6572

Ce document vous est offert par Université Rennes 2

\section{Référence électronique}

Stéphane Héas et Omar Zanna, "L'exercice sportif

entre douleur et plaisir », Revue des sciences sociales [En ligne], 53 | 2015, mis en ligne le 30 juin 2018, consulté le 03 juillet 2019. URL : http://journals.openedition.org/revss/2858 ; DOI : 10.4000/ revss. 2858 


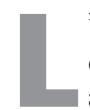
'analyse des pratiques sportives de compétition ${ }^{1}$ révèle une ambivalence (Bodin, Robène, Héas, 2004; Young, 2004, etc.). Ces pratiques génèrent, en effet, de la violence tout autant qu'elles la contrôlent, si ce n'est la réprouvent. L'approche éliasienne demeure particulièrement éclairante pour saisir cette gouvernance intime et sociale du sport quand bien même elle est critiquée à la fois en termes de corpus mobilisés (Duerr, 1997) et pour son ethnocentrisme sous-jacent (Bodin, Robène, 2014), si ce n'est son européocentrisme. Reste l'indication historique d'un processus au long cours de structuration et in fine d'euphémisation des relations humaines dans les sociétés contemporaines; ce que $\mathrm{N}$. Élias a démontré dans La civilisation des moeurs et La dynamique de l'Occident ${ }^{2}$. Le seuil social d'acceptation de la violence sur soi ou sur autrui a considérablement baissé. Logiquement la douleur physique n'est plus autant l'objet d'une valorisation en dehors d'espaces-temps spécifiques comme la guerre ou le sport justement. Cet adoucissement des mours pour reprendre Élias per- met de mieux cerner l'intérêt d'interroger les activités sportives en tant qu'activités culturelles particulières de ce point de vue.

D'autres approches de la douleur ont été mobilisées, notamment celle qui précise les liens privilégiés entre douleur et souffrance dans et par les rapports au corps dans le cadre d'activités liées au risque et/ou avec des effractions cutanées susceptibles de perturber les interactions sociales (Le Breton, 1990, 1991). Une approche complémentaire, reprise en partie ici soutient que la douleur générée par les pratiques sportives constitue un support d'éducation à l'empathie (Zanna, 2008, 2010). Le sens de la douleur pour soi et pour les autres, dans le contexte sportif, devient alors essentiel à mieux saisir.

Au-delà des controverses scientifiques toujours actives, l'ambivalence sportive est inscrite dans le rapport des pratiquant.e.s à leur corps simultanément sacralisé et persécuté (Détrez, 2002), tantôt source de douleur, tantôt source de plaisir. L'analyse des discours de jeunes sportifs français et espagnols (Bodin et al., 2013) met en exergue cette ambivalence qui 
se déploie dans une quadrature de rapports à la douleur, au soin, à la performance et, éventuellement, dans le recours au dopage. Nos réflexions prolongent les résultats de cette recherche qualitative, non sans avoir au préalable précisé l'enjeu de la douleur et de sa légitimité dans le domaine des sports aujourd'hui.

\section{Les douleurs vitales}

La douleur ne peut plus être considérée uniquement dans son versant négatif (Zanna, 2010). Elle apparaît également comme une précieuse compagne tout au long de la vie (Annequin, 2002). «Au cours des premières années de la vie, les petites douleurs de tous les jours participent à de nombreux apprentissages" (FournierCharrière, 2007, 34-35). En ce sens, la douleur aide à la connaissance des limites du corps et de ses capacités. Les activités physiques et sportives entrent directement en action à ce niveau d'apprentissage par corps. Prendre des coups dans un combat de boxe, frotter son corps aux autres dans une mêlée de rugby, se couronner le genou ou le coude à la suite d'une chute de vélo ou de roller, se cogner la tête contre une poutre en gymnastique, ressentir un violent "point de côté » en course à pied, etc., ces douleurs sportives font partie d'un apprentissage structurant (Gauvain-Piquard \& Meignier, 1993). Surtout, sans le ressenti de la douleur, notre vie serait à chaque instant menacée. A contrario, à un niveau anthropozoologique plus large, «le rôle crucial de la douleur comme signal d'alarme contribuant à la survie de l'espèce, l'enracinement de sa signification symbolique dans le registre religieux, la crainte qu'elle inspire à tout être humain comme marque de sa vulnérabilité, donnent en effet à la représentation d'un homme sans douleur une connotation à la fois magique et inquiétante» (Danziger, 2010, 47). Privée de la capacité à ressentir la douleur, l'existence humaine deviendrait terriblement fragile, accroissant encore sa vulnérabilité. "La douleur contraint à l'apprentissage lucide et malaisé des dangers qui menacent l'intégrité physique. [...] Vecteur de l'éducation de l'enfant, en sanctionnant aussitôt toute action inappropriée de sa part, elle enseigne la prudence nécessaire qui compense la fragilité native de la condition humaine» (Le Breton, 1995, 14-15).

La douleur est donc une précieuse compagne, en ce sens qu'elle conduit les individus à prendre conscience de leur corps. Cette conscience surgit habituellement lorsque le silence des organes est rompu. Des techniques de relaxation et/ou de concentration particulière permettent une prise de conscience corporelle en dehors des phases douloureuses (Héas, 2004). Elles sont désormais largement utilisées dans les phases de préparation, d'action et de récupération des dépenses physiques sportives. En définitive, la douleur en tant que sensation est l'occasion d'éprouver la vie en soi. Et ce pouvoir, nous le tenons du corps lui-même, de son mode fondamental de pâtir: la douleur. Pas de douleur «sans corps pour l'éprouver. Pas d'angoisse, pas de plaisir, pas de désir même sans corps" (Dejours, 2001, 153). La douleur permet aux barrières corporelles et psychiques de s'instaurer. «Elle estle signal d'un désordre quelque part dans le corps ou d'une injure subie qu'il convient de faire cesser et de réparer » (Vincent, 1986, 214).

Signal d'alarme, la douleur s'apparente donc à une compagne qui enseigne à l'individu et comme nous allons le voir, au sportif jusqu'où il peut (ne pas) aller. Cette douleur, les jeunes athlètes rencontrés dans le cadre de la recherche comparative menée en France et en Espagne (Bodin et $a l ., 2013^{3}$ ) en parlent comme un mal légitime et nécessaire pour prétendre à l'excellence sportive.

\section{Douleurs légitimes et légitimées}

La gestion de la douleur lors des entraînements ou bien des compétitions participe de la pratique de nombre de sports. Elle concourt à la formation d'un être humain aux prouesses élevées, si ce n'est exceptionnelles (Héas, 2010, 2011). Si la douleur accompagne tout individu de toutes les civilisations, ce qui diffère d'une société ou d'un groupe d'individus à l'autre, ce sont l'appréhension, le traitement et le sens même de cette douleur ressentie personnellement ou provoquée chez l'autre (Le Breton, 1997; 2010).

Ces expériences de la douleur constituent donc des révélateurs culturels mais aussi sociaux puisque les attendus sociaux ne valorisent pas de la même manière les contacts, les chocs susceptibles de l'engendrer. La logique même des sports est variable et n'expose pas les corps des pratiquant.e.s aux mêmes éventualités ou risques de douleur. Les risques encourus en boxe ne sont, en effet, pas de même nature que ceux d'une course de demi-fond. À l'évidence, en fonction de la discipline sportive, la quête de la performance n'implique pas les mêmes engagements corporels. Cette variation pose d'ailleurs des problèmes d'image et de représentation collective à certaines activités physiques. Citons les déboires des différentes formes de combat sans limite (ultime fight) qui effraient par les engagements extrêmes parfois jusqu'à la mort des pratiquant.e.s, ou d'un autre côté la suspicion de non-dépense physique chez les cavaliers puisque le cheval est parfois considéré comme le seul athlète (Régnier, 2014). Or, ces engagements participent - chez les plus jeunes à des rituels (souvent douloureux) censés aider à construire leur identité alors que leurs coordonnées sociales sont encore en construction. Les jeunes garçons sont particulièrement concernés par ces rites symboliques de passage où le don de soi - qui s'observe parfois par une oblation de sang est un indicateur d'engagement plein et entier dans ce processus de devenir Autre. C'est pourquoi, ce qu'un regard extérieur cursif appréhenderait comme un agir violent, est davantage à considérer comme éléments d'un rituel destiné à accompagner et valider un changement de statut.

Les ressources ethno-anthropologiques concernant les rites de passage, les (auto)mutilations au sein de socié- 
tés dites traditionnelles permettent justement d'établir une proximité avec les «cultures sportives» contemporaines et occidentales (Van Gennep, 1981; Goguel d'Allondans, 2002). Les propos de Pierre Clastres, décrivant le rite de passage à l'âge adulte, qui consiste à inciser en profondeur le dos du haut de l'épaule au sommet des fesses, avec une pierre choisie pour l'occasion, sont à cet égard éclairants pour comprendre la place centrale de la douleur dans le changement de statut: "Pas plus que pendant l'imbi $m u b u$ [rite de perforation de la lèvre inférieure des jeunes garçons âgés de 15 ans] on entendra le jeune laisser échapper plaintes ou gémissements: plutôt perdra-t-il connaissance, mais sans desserrer les dents. À ce silence se mesurent sa vaillance et son droit à être tenu pour un homme accompli" (Clastre, 1972, 142). Puisque les rites de passage sont sans doute aujourd'hui plus diversifiés que par le passé, les jeunes sportifs sont d'une manière différente eux aussi engagés dans ce type de passage qui transforme leur statut. Mais au rite de passage unique et collectif se substitue désormais des passages plus individualisés, et surtout multipliés dans des sphères parfois différentes et avec des groupes eux-mêmes différents. Les groupes de pairs peuvent aujourd'hui se succéder, mais plus encore se cumuler. L'engagement sportif peut ne constituer qu'un type particulier d'engagement parmi d'autres à un moment d'une trajectoire. Reste que lorsque l'engagement sportif est exigé 4 comme dans le cas des jeunes sportifs inscrits dans des centres de formation ou des "pôles Espoirs», tremplins des sélections nationales par exemple, le rite se durcit en quelque sorte. La "marche» à franchir peut s'avérer trop haute. On comprend mieux alors pourquoi certains jeunes athlètes rencontrés refusent plus ou moins consciemment de s'engager pleinement dans une carrière sportive, surtout après des déconvenues physiques, comme les blessures à répétition par exemple vécues par eux-mêmes ou leurs camarades.

Comme nous avons pu l'observer au cours de cette enquête, les différentes expériences sportives relèvent un rapport ambigu avec la douleur. Dans le milieu sportif, la résistance à la douleur est souvent perçue comme le gage d'une bravoure susceptible de conférer à celui/celle qui en fait l'épreuve une (prétendue) disposition exceptionnelle et consubstantielle au statut d'athlète accompli et reconnu dans le groupe. Obsédés par le progrès et la performance, les jeunes athlètes (et leurs cadres!) prétendant au haut niveau vantent ce rapport à la douleur comme un viatique pour accéder à la plus haute marche du podium. Cette incorporation d'un rapport légitimé et légitimant de la douleur devient, ainsi, une véritable disposition de leur identité sportive d'autant plus cultivée et (dé)montrée de manière plus ou moins ostentatoire chez les sportifs en devenir. Pour ces jeunes, donner à voir aux autres - aux plus confirmés notamment - leurs capacités à «dolorir $^{5}$ ", soit expérimenter une douleur, sur le terrain de la pratique s'apparente à une forme de «socialisation anticipatrice». Les individus désirant une affiliation dans un groupe ont, en effet, "toujours tendance à partager les sentiments et à se conformer aux valeurs du noyau le plus prestigieux et respecté du groupe. Le conformisme entraîne l'acceptation par le groupe et vice versa» (Merton, 1957, 216). L'enjeu d'une rencontre sportive est d'ailleurs souvent l'occasion d'exacerber cette concurrence qui se donne à voir par un engagement physique paroxystique où la violence infligée aux corps est repérable au travers des mimiques, des rougeurs, des pâleurs, des timbres de la voix, des postures plus ou moins maitrisées... Les manifestations corporelles de la douleur ne trompent pas, car, dans de tels cas, le corps n'est pas qu'un corps il est également langage qui parle à l'observateur (Descamps, 1966; Zanna 2015).

Les jeunes sont d'autant plus prompts à participer pleinement à ces mises en jeu du corps que le risque peut permettre justement d'acquérir une valeur supplémentaire, sur le modèle de l'ordalie ${ }^{6}$. Logiquement, dans cette confrontation aux limites, l'idée non du «mal», mais de se «faire mal» est valorisée au point d'être devenu l'amer du sportif accompli. Dans ce contexte, la douleur confère une légitimité dès lors qu'elle est apprivoisée puis systématisée et par conséquent naturalisée, pour devenir finalement une véritable disposition de l'identité sportive. Ce processus s'inscrit dans un apprentissage dont les étapes si elles varient selon les disciplines restent caractérisées par une quête du plaisir dans la douleur, en particulier celle liée à l'entraînement et ses exigences. En témoignent les propos de cette cycliste de 18 ans:

«Si tu as la possibilité de plus te faire aussi mal, je pense que c'est ça, parce qu'après si t'as jamais touché et en soi moi j'aime le fait de se faire mal, même si c'est horrible sur le coup... sinon tu nes pas une vraie cycliste [...] Ouai, je pense qu'il faut être fort ${ }^{7}$ mentalement pour faire du vélo presque aussi fort mentalement que physiquement parce que psychologiquement c'est dur. Ben t'es fière de toi après, t'as réussi, t'as pas abandonné, voilà c'est ça et puis c'est agréable...»

Q: Est ce qu'il y a beaucoup de gens à abandonner?

Ben ça dépend de ton état d'esprit quoi, si parce qu'il $y$ en a quand ils sont petits ils marchent sans se faire mal, ils sont quand même au-dessus des autres, donc quand tu grandis et que tu deviens moins fort que les autres et que tu nas pas appris à te faire mal, tu arrêtes. Mais comme depuis le départ tu t'es fait mal et tu continues, ce n'est pas la défaite qui fait [...] faut apprendre à continuer à se battre.»

Les processus de socialisation et la formation d'une véritable disposition à l'acceptation de la douleur générée par la mise à l'épreuve des métabolismes (acidité cellulaire, crampes, courbatures, trachée irritée...) et atteintes extérieures au corps (blessures) du jeune sportif pointent également la place et le rôle de l'entourage (Mennesson, 2005; Zanna, 2010). Si le rôle de l'entraîneur consiste à veiller si ce n'est à la bonne santé des athlètes du moins leur intégrité, de fait il est là pour enregistrer et parfois valider les douleurs ressenties:

"Ça dépend des jeunes, jen ai une qui n’est jamais blessée, ça fait deux ans que je suis avec, elle na jamais été blessée, je ne vais pas dire les petits pépins, les petites douleurs qui hop le lendemain sont terminées. » (Entraîneur d’athlétisme). 
Vivre des expériences avec seulement des "petits pépins» devient un sésame inespéré, tant la casse sportive est importante surtout dans le cadre de la recherche de performance. Un véritable apprentissage de la douleur est donc nécessaire au devenir sportif. Observée de l'extérieur, cette socialisation spécifique s'apparente à une potentielle "carrière " déviante quand bien même elle est rarement perçue et étiquetée comme telle. Elle est bien plus souvent considérée comme une réponse hyper-conforme à la culture sportive. Ce qui nous amène à penser différemment ce rapport discipliné au corps dans le cadre sportif (Hughes, Coakley, 1991).

\section{Les douleurs nécessaires}

L'apprentissage sportif met non seulement à mal l'équilibre homéostatique mais mobilise également des états corporels limites au cours duquel la douleur apparaît comme un «symptôme sportif» à percevoir, pour ensuite apprendre à en apprécier les effets et en définitive à générer du plaisir. Dans cette socialisation sportive, la douleur, les courbatures et autres sensations s'apparentent à ce qui subsiste du rêve au moment du réveil: quelque chose de non élaboré. Reste alors, pour les athlètes qui se prêtent à l'exercice - in fine au rituel-, à faire le chemin qui va de l'expérience (ressentie et partagée) de la douleur et des émotions à la révélation et la reconnaissance des autres athlètes comme des semblables, c'està-dire des autres soi-même (Ricoeur, 1990). Cet apprentissage groupal peut être repris dans le contexte sportif en termes de déviance positive et de l'hyper-conformité sportive (Hughes, Coakley, 1991).

Ce processus a été décrit par Becker (1963) pour rendre compte de la carrière des fumeurs de marijuana. Apprendre à avoir bien mal comme à bien planer, apprendre à apprécier des sensations corporelles aux premiers abords désagréables participe de ce processus qui conduit mutatis mutandis à devenir expert et un pair dans l'art de fumer pour les uns et dans la discipline sportive pour les autres. Aux dires des sportifs en devenir la douleur - toujours minimisée - est un mal nécessaire pour prétendre à cette forme d'adoubement, toujours silencieux, dévolu par les pairs devenus experts:

"Bah c'est... si je ne sens pas... enfin normalement si on a mal on le dit... mais comme jai jamais mal, et bien non je ne dis rien, je ne sens pas" (Basketteuse, 15 ans) "J'ai continué à forcer sur mon genou et puis ma rotule s'est abîmée et puis... et puis voilà, c'est trop super, mais bon, y en a pour deux semaines, deux mois pardon de repos donc» (Basketteuse, 21 ans).

Les lapsus ici sur les temps d'arrêt forcé sont révélateurs d'une temporalité sportive cadrée et programmée chaque année des mois à l'avance. La moindre interruption complique cette planification des forces sportives en présence:

"En général enfin... en général on reprend plus tôt que... que ce que prévoient les médecins généralistes ou... même les médecins du sport» (Basketteuse, 17 ans)

Face à cette expérience d'une douleur, voire d'une blessure jugée nécessaire à la socialisation/sportification/ sportivisation (Lebreton et al., 2010), de nombreuses "recettes", véritables «ficelles du métier» (Becker, 2002) sont échangées. Elles permettent à la fois de mieux vivre sa vie de sportifs, et de maintenir une activité et une performance attendue. Elles révèlent aussi la nécessité pour beaucoup de sportifs de "composer» avec la douleur, de s'adapter et de se mettre en quête de solutions dites "miracles».

"Et les sportifs entre eux, il y a plein de gens qui donnent des conseils et tout. Eh bien, c'est Jimmy Casper un professionnel qui ma donné une marque de pansement et c'est vrai que ça a été miracle.» (Jeune cycliste, 17 ans)

Une question se pose dès lors: jusqu'où ces sportifs sont-ils prêts à aller? Contrôle de soi, du corps, de la douleur, de la blessure, autocontrôle des émotions et gestion du stress, peuvent amener le sportif à la rupture.

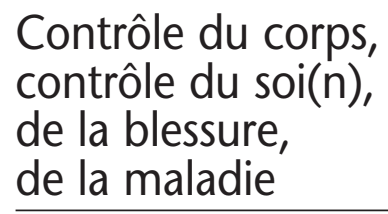

Nombreux sont les propos recueillis qui précisent que face au rythme d'entraînement et d'enchaînement des compétitions, "le corps dit stop (dixit)». Cette évocation récurrente n'est pas propre aux sportifs. Elle est largement partagée, voire valorisée, dans les sociétés contemporaines notamment par certains secteurs professionnels, économiques ou sociaux comme les professions médicales (Le Breton, 1999). Elle reflète une représentation dualiste de l'être humain, avec d'un côté l'esprit (le mental, la motivation, etc.) et de l'autre le corps (courbatures, plaisirs, douleurs, etc.). Cette conception émique d'un corps alter ego, n'est, rappelons-le, pas le propre des sportifs, qu'ils soient jeunes ou non. Ce dualisme objectivant le corps, le séparant de l'être humain a historiquement permis des progrès médicauxincontestables; cependant, il conforte une séparation qui n'est pas fonctionnelle, pas réaliste. Elle comporte un risque de scission entre des "éléments» distingués pour l'analyse à plat, anatomique, de l'être humain (Le Breton, 1993).

Les sports et notamment les gymnastiques (Ulmann, 1977) ont utilisé et même abusé de ces distinctions pour mettre en place des exercices calibrés, standardisés, et de ce fait aisés à mettre en œuvre dans les écoles, les clubs sportifs. Mais ce découpage de l'être humain en partie, plus ou moins étanches, ne reflète absolument pas la réalité. Surtout, les interactions entre le corps et l'esprit sont permanentes dans la réalité quotidienne (Varela, 1993). En effet, l'expérimentation du monde, par le biais du corps, favorise autant la compréhension que l'esprit seul. Christophe Dejours (2001) ne précisait-il pas que, pour advenir, le corps doit se heurter à la résistance du monde. Tout bien considéré, Descartes a commis une erreur (Damasio, 1995) et Spinoza avait raison (Damasio, 2003). "Spinoza a eu l'intuition 
du dispositif anatomique et fonctionnel global que le corps doit mettre en ouvre pour qu'apparaisse l'esprit avec lui ou, plus précisément, avec et en lui. [...] l'esprit et le corps sont des processus parallèles et mutuellement corrélés [...] Ils se doublent l'un l'autre en tous endroits, comme deux faces d'une même chose" (Damasio, 2003, 210-217). Une relation indissociable existe donc entre émotions, sentiments, conscience et raison, c'està-dire entre corps et esprit.

Plus encore, cette dépossession du corps propre du "sportif en devenir" est à mettre en lien avec la dépossession plus globale qu'il subit dans le cadre d'une éducation sportive qui le plie à des adultes qu'ils ou elles soient cadres, animateurs, dirigeants, entraîneurs, professeurs, etc. Cette dépossession corporelle et psychique est inscrite dans des emplois du temps souvent chargés combinant activités scolaires et périscolaires, dont le sport. Les consignes, les exercices routiniers et souvent quotidiens sont tous orientés vers l'accomplissement d'une tâche sportive. L'intériorisation et le «jeu» risqué de ces contraintes sont explicites:

"Quand je suis blessé je vais voir le kiné, et il me dit combien de temps j'ai d'arrêt et j'essaye de minimiser quoi, pour reprendre le plus vite possible» (Basketteur, 16 ans)

L'appel aux ressources médicales et paramédicales est permanent; il est toujours adapté aux conditions sportives du moment. Le planning des activités primant systématiquement sur celui du médical. In fine, tout est organisé pour que le poids de la décision revienne à l'individu alors même que c'est le système sportif qui produit et maintient la pression temporelle. À l'évidence, dans le monde sportif comme dans celui de l'entreprise, l'autonomie est devenue une méta-valeur prisée. Les "desseins temporels» contemporains sont, en effet, de plus en plus individualisés et soumettent l'individu à une course à l'autonomie toujours plus affirmée (De Gaulejac, 2011). Mais à y regarder de plus près, l'autonomie des jeunes sportifs, apparaît moins importante tant le système fédéral notamment subvient à leurs besoins sportifs ou non:
"Comment je gère? Eh bien, je fais attention, jécoute les conseils de la kiné, je me soigne. Après, au niveau de la planification par le coach, il évite le travail qui peut aggraver ce genre de problème.» (Athlète, 20 ans)

Les propos nombreux autour de la thématique de la blessure et de sa gestion apparaissent comme une nouvelle ambivalence qui replace le corps au centre de la décision. Or, l'apprentissage de la domestication de la douleur vient en contradiction avec l'idée de laisser parler le corps. Le jeune sportif n'assume pas de devoir se reposer et continue le rythme des entraînements imposés. Dans cette course à la performance attendue par le système sportif, seule la blessure constitue une justification légitime à l'arrêt de pratique: "Elle se fait une méga entorse le soir, la veille du match décisif. Bon elle était sur le banc strappée, je l'ai laissé un petit peu au début, puis on a vu que ça allait le faire alors elle a serré les dents et elle a joué, je ne l'aurai pas fait avec une jeune et elle a joué parce que ce n'était pas pour faire des miracles. C'était que pour que psychologiquement ses copines sachent qu'elle était là.» (Un entraîneur de basket)

Si effectivement des positions différentes s'expriment, concernant l'arrêt du sportif et la gestion de la reprise de l'activité physique, elles mettent toujours a minima en évidence un ascendant, une distribution de la gestion de ce corps et avec elle du processus de décision, dans l'économie et la politique des corps où le pouvoir sur le sportif ou la sportive est toujours activé. Ce protocole est décrit par un autre entraîneur en ces termes:

"On a instauré un protocole, le joueur est malade, toute la semaine il est avec nous, il est malade, il va voir le médecin du club, qui prend le relais de son médecin traitant, voilà. Le joueur se blesse, et bien on doit l'arrêter, suivant ça on le fait suivant la gravité entre guillemets de la blessure, souvent c'est un peu de traumato donc c'est un peu de kiné en priorité, il voit le kiné et ceest le kiné qui nous donnera le feu vert s'il doit reprendre. Si c'est un truc plus grave, il va voir le médecin, le médecin lui fait passer radio, IRM, si possible dans les $48 \mathrm{~h}$, parce qu'on arrive à avoir des IRM très rapides, avec notre médecin donc c'est intéressant.
Et par rapport à ce protocole-là, c'est toujours le médecin, kiné qui donnera le feu vert, pour faire reprendre un joueur. Moi je ne fais pas reprendre un joueur si le kiné na pas donné son feu vert pour le faire jouer sur un week-end. Mais les jeunes ici ont des conventions ou des contrats stagiaires, ou aspirants, donc il y a des pouvoirs de délégation qui sont donnés au niveau de la structure concernant le médecin ainsi de suite, donc en fait ils suivent le protocole quion leur donne.»

La médecine du sport agit ici à plein. Sous couvert d'un suivi médical rigoureux, l'engrenage répétitif des compétitions incite tous les partenaires à ne pas respecter les temps de repos, les temps de récupération, les temps de cicatrisation. L'efficience sportive est obtenue alors au prix de la santé des athlètes qui doivent tenir coûte que coûte pour garder leur place, pour pouvoir espérer atteindre les objectifs, individuels mais aussi collectifs.

\section{La division du travail corporel dans le cadre de la discipline sportive}

Une nouvelle ambivalence peut être ici mise en évidence. L'obsession corporelle observée par les sportifs enquêtés à travers l'abandon de ce corps à la douleur, voire à la blessure, redouble avec le fait que ces jeunes en confient la (p)réparation finalement au champ médical (kinés, médecins, et divers autres thérapeutes). Sous certains aspects et à des moments clés de la carrière, le corps du sportif, lui est pour ainsi dire confisqué au profit d'une prise en charge totale. A ce moment-là de sa carrière, sa socialisation s'apparente à une "socialisation secondaire totale»(Zanna, 2003). Il est soumis à une discipline rigoureuse, voire coercitive, dans la gestion de son corps, de son emploi du temps, de son régime alimentaire et parfois même dans ses relations amoureuses. À un premier niveau s'organise un véritable maillage du travail sur le corps du sportif repérable dans la connaissance de son propre corps de la part du jeune sportif lui-même: 
"L'avantage de commencer tôt ce genre de sport c'est qu'on apprend à bien se connaître. Je sais très bien que je n'irai pas courir jusqu'à claquer quoi. Je sais où est ma limite en fait.» (Athlète, femme, 21 ans)

Au-delà de simples sensations corporelles, se développent des connaissances, pour ne pas dire une expertise de la douleur, de son sens, de ses conséquences et d'une manière plus globale de l'usage du corps. Cette connaissance est perceptible dans les discours de ces jeunes notamment dans l'usage récurrent du vocabulaire médical ${ }^{8}$ (Douguet, 2000). Cette appropriation de ce vocabulaire concerne plus précisément les pathologies et les blessures, la maîtrise du protocole de soin (aussi prescriptif soit-il), l'anticipation parfois du diagnostic, et parfois du traitement:

"Est ce que tu as été blessée souvent?» "Non pas souvent. Récurrent tous les ans, tous les étés à cause des haies, jai des périostites, ce sont des inflammations au niveau du tibia. L'année dernière, j’utilisais des bandes de strap à n'en plus compter à tous les entraînements. Et là en fait, je me suis blessée, enfin on ne peut pas appeler ça vraiment une blessure parce que je me suis fait ça mardi. Ça faisait plusieurs fois que javais mal au quadriceps, je me suis fait ça pendant une séance de sprint que javais fait en rentrant, pour regagner de la vitesse.» (Jeune fille, athlète, 17 ans)

Cette appropriation concerne l'anatomie, ce qui semble somme toute logique puisque les jeunes sont directement confrontés aux discours des adultes, et en l'occurrence ici des experts consultés en cas de blessures. Mais ce type d'adhésion concerne également la connaissance du réseau médical au niveau local, au moins.

Tout se passe comme si cette culture médicale redoublait la culture somatique des jeunes. Pour autant, ils ne semblent pas en mesure d'éviter les blessures. Elles sont en effet jugées nombreuses: tous les athlètes rencontrés se souviennent de blessures, plus ou moins graves. Cette fréquence du sport iatrogène est associée inévitablement à ce qu'ils se représentent comme les plus mauvais souvenirs de leur carrière. Par conséquent, si la douleur est gérée, et parfois minimi- sée, elle n'est pas pour autant valorisée en elle-même en tous les cas dans le cadre a posteriori des entretiens qui incitent les jeunes à se "plonger» dans leurs propres expériences passées.

Le contrôle des corps est notamment présent en période de compétition où «l'affûtage» nécessite perte de poids et gain de muscles: dialectique que les pratiquants de sport de combat connaissent bien. Du contrôle, il en est également question à propos de l'hygiène et la gestion du sommeil lors des stages de préparation en centres de formations. Là encore, les corps sont (sou)mis à l'épreuve. Cette surveillance panoptique des corps est d'autant plus marquante qu'elle a cours à l'adolescence, lorsque, paradoxalement, l'individu tente de s'individuer, de faire son baptême de l'ailleurs...

Le jeune sportif, en passe vers le haut niveau, vit dans un entre-deux, un espace liminaire où les engagements corporel, social et psychologique sont entiers. Le sport de haut niveau est, en effet, exclusif! Les stratégies d'évitement de ces contraintes existent, mais peuvent difficilement être explicitées et racontées à un enquêteur de passage. Là, comme dans le domaine du dopage, la parole donnée à l'extérieur de la sphère sportive se doit d'être restreinte au strict minimum. Nous avons à plusieurs reprises repéré cette retenue au cours des entretiens. Les sourires en coin, les phrases stoppées net ont été légion. Et, il suffit de tendre l'oreille pour comprendre «ce que parler ne veut pas dire» (Bourdieu, 1982). Peut-on parler d'acquisition d'une certaine "sagesse» ou bien ce processus relève-t-il plutôt d'un assujettissement plus profond encore? Tout bien considéré, il semble s'opérer une délégation, voire un abandon de ce corps dont le sportif a la maîtrise et l'expérience.

\section{La blessure qui dure pour conclure avec le terrain}

Une rupture surgit néanmoins dans cette distribution de la propriété corporelle de la performance dès lors qu'il y a incident. Avec la blessure et finalement les retards dans la performance voire les contre-performances associées, la responsabilité incombe subitement au sportif, ou plus exactement à son corps improductif. Le sportif (re) devient dès lors responsable de "sa blessure». La responsabilité n'est en effet que très rarement partagée:

"Et on voit bien! Là, mon corps a besoin de se reposer, de se remettre en condition; mon entraîneur essaye de me faire faire un truc, je ne peux pas physiquement... Donc ce n'est pas moi qui demande du repos, ni mon entraîneur, c'est vraiment le corps qui dit «moi jen peux plus, je sature donc faut me ménager et après on pourra reprendre de manière sérieuse mais là je ne peux plus.» (Athlète, 19 ans)

$\mathrm{Ne}$ plus pouvoir agir comme un sportif revient à rompre concrètement avec le terrain sportif, avec un risque toujours présent de ne plus retrouver sa place. Aux yeux de ces jeunes l'arrêt sur blessure est susceptible de les condamner à une régression difficilement récupérable. Cette focale sur l'interruption comme source de dégradation sportive n'est pas sans rappeler dans le milieu professionnel les absences pour cause d'accidents du travail ou bien d'accidents de la vie courante. Or, là encore les jeunes même et surtout parce qu'ils s'en soucient peu, et aussi par manque d'expérience, sont largement victimes d'accidents (OCDE, 2007; Lydie, 2008).

\section{Bibliographie}

Annequin A. (2002), La Douleur chez l'enfant, Paris, Masson.

Becker H. (1985, tr. fr.), Outsiders. Etudes de sociologie de la déviance, 1963, The Free Press of Glencoe.

Bellay J., Héas S. (2011), «Les dermatologiques sportives: essai de comparaison entre natation et judo ", ACAPS, Rennes, 24-26 octobre.

Bodin D., Robène L., Héas S. (2004), Sports et violences en Europe, Strasbourg, Editions du Conseil de l'Europe, août.

Bodin D. (dir.) (2013), Se doper ou pas. 28 préconisations pour prévenir le dopage chez les jeunes sportifs en Espagne et en France formalisées à 
partir d'une approche multidimensionnelle des processus de conduites dopantes, Rapport final pour l'AMAWADA, 365 pages.

Bodin D., Robène L. (2014, à paraître). «Sport et civilisation. La violence maitrisée. De l'indéfinition de la violence à l'Illusion pacificatrice des sports modernes", International Journal of History of Sport.

Bourdieu P. (1982), Ce que parler veut dire. Paris, Fayard,

Burnay N., Ertul S., Melchior J. Ph. (dir.) (2013), Parcours sociaux et nouveaux desseins temporels, Louvain-la-Neuve, Academia.

Chirpaz F. (1977), Le Corps, Paris, PUF.

Clastres P. (1975), La Société contre l'Etat, Paris, Editions de Minuit.

Clavandier G. (2009), Sociologie de la mort. Vivre et mourir dans la société contemporaine, Paris, Armand Colin.

Cuin Ch. H. (2011), Durkheim: modernité d'un classique, Paris, Editions Herman.

Damasio A.R. (1995), L’Erreur de Descartes. La raison des émotions, Paris, Odile Jacob.

Damasio A. R. (2003), Spinoza avait raison. Joie et tristesse, le cerveau des émotions, Paris, Odile Jacob.

Danziger N. (2010), Vivre sans la douleur?, Paris, Odile Jacob.

Dejours Ch. (2001), Le Corps d'abord, Paris, Payot.

Détrez C. (2002), La construction sociale du corps, Paris, Seuil.

Dolto F. (1990), Paroles pour adolescents ou le complexe du homard, Paris, Hatier.

Duerr H. P. (1997), Nudité et pudeur, Editions de la Maison des Sciences de l'Homme.

Ferez S., Thomas J. (dir.) (2012), Sport et VIH. Un corps sous contrainte médicale, Paris, Editions Téraèdre.

Fournier-Charrière É. (2007), «La douleur laisset-elle des traces chez le bébé?», Spirale, n 42 , p. 34-35.

Gaulejac de V. (2011), Travail, les raisons de la colère, Paris, Seuil.

Gauvain-Piquard A., Meignier M. (1993), La Douleur de l'enfant, Paris, Calmann-Lévy.

Goguel d'Allondans T. (2002), Rites de passage, rites d'initiation: lecture d'Arnold van Gennep, Canada, Les Presses de l'Université Laval.

Héas S., (2004). Anthropologie des méthodes de relaxation, Paris, L'Harmattan.

Héas S., Bodin D., Amossé K., Kerespars S., (2004). "Football féminin: "C'est un jeu d'hommes" ", Cahiers du Genre, n 36, p. 185-203.

Héas S. (2010), Les virtuoses du corps. Enquête auprès d'êtres exceptionnels, Paris, MaxMilo, collection Essai/document, août.

Héas S. (2011), À corps majeurs. L'excellence corporelle entre expression et gestion de soi, Paris, L'Harmattan, Collection Le Corps en question (grand format), décembre.

Hughes R., Coakley J. (1991), «Positive Deviance among athletes: the implications of overconformity to the sport ethic", Sociology of Sport Journal, $n^{\circ}$ 8, p. 307-327.
Kotobi Henri K., (2009). Le dualisme du corps et de l'esprit à l'épreuve de la douleur: Qu'est-ce que la douleur?, vol. 2, Paris, L'Harmattan.

Lebreton F., Routier G., Héas S., Bodin D., (2010). "Cultures urbaines et activités physiques et sportives. La «sportification» du Parkour et du street golf comme médiation culturelle», The Canadian Review of Sociology and Anthropology, vol. 47, n³, p. 293-317.

Le Breton D. (1991), Passions du risque, Paris, Métailié.

Le Breton D. (1993), La Chair à vif. Usages médicaux et mondains du corps humain, Paris, Métailié.

Le Breton D. (1997), Anthropologie de la douleur, Paris, Métailié.

Le Breton D. (1999), L'adieu au corps, Paris, Métailié.

Le Breton D., (2010). Expériences de la douleur: entre destruction et renaissance, Paris, Métailié.

Lydie V. (2008), Le suicide des jeunes. Mourir pour exister, Paris, Syros.

Mennesson C. (2005), Être une femme dans le monde des hommes: socialisation sportive et construction des genres, Paris, L'Harmattan.

Merton R.K. (1957), Éléments de théorie et de méthode sociologique, Paris, Armand Colin, 1997.

Meudec M. (2007), Le kout poud: maladie, vodou et gestion des conflits en Haïti, Paris, L'Harmattan.

OCDE (2007), Des emplois pour les jeunes/Jobs for Youth, Belgique, Edition OCDE.

Régnier P., (2014). Devenir cavalier: une expérience d'apprentissage par corps: essai de socioanthropo-zoologie des pratiques et techniques équestres, thèse de sociologie, dirigée par $\mathrm{S}$. Héas et M. Calmet, soutenue le 9 janvier, université de Rennes 2.

Robène L., Bodin D. (2014, à paraître), Sports et violences au miroir des sociétés modernes. Les ambiguïtés sportives. International Journal of History of Sport.

Van Gennep A. (1981), Les Rites de passage. Etude systématique des rites..., Paris, Éditions Picard.

Varela F., Thompson E., Rosch E. (1993), L'inscription corporelle de l'esprit, Paris, Le Seuil.

Vincent J.-D. (1986), Biologie des passions, Paris, Odile Jacob.

Young K. (2004), Sporting Bodies, Damaged Selves: Sociological Studies of Sports-Related Injury, Elsevier.

Zanna O. (2008), «L'expérience partagée de la douleur physique peut-elle être socialisante?", Revue internationale du sport et de l'éducation physique, ${ }^{\circ} 79$, p. 95-112.

Zanna O. (2010), Restaurer l'empathie chez les mineurs délinquants, Paris, Dunod.

Zanna O., Lacombe Ph, (2003), "Anthropologie du geste sportif en milieu carcéral», Agora débats/jeunesses, $\mathrm{n}^{\circ} 33,4^{\mathrm{e}}$ trimestre.

Zanna O. (2015), Apprendre par corps l'empathie à l'école: tout un programme?, Revue Science de l'éducation.

\section{Notes}

1. Une première version de cette analyse a été publiée dans le rapport AMAWADA (Sempé G., Héas S., dans: Bodin et al., 2013, 136-176).

2. Titre de la traduction française de sa thèse écrite, rappelons-le, en 1939 en langue allemande.

3. Ce travail comparatif a débouché sur 28 préconisations visant à prévenir le dopage chez les sportifs les plus jeunes.

4. Indiquons ici que ces formations sportives sont des espaces de formation adultocentrés: les exigences de ces institutions sportives prennent souvent les contours d'une contrainte totale avec un contrôle des corps, des temps, des espaces, etc.

5. Indiquer ici «souffrir » reviendrait à négliger la différence entre la souffrance et la douleur.

6. L'ordalie est une épreuve censée définir le destin lors d'une situation critique telle une maladie.

7. Notons, et c'est un classique de la sociologie des sports, que souvent les sportives pratiquant une activité traditionnellement masculine, omettent le «e» et plus largement ne pensent pas à féminiser leurs phrases (Héas et al., 2004).

8. Un phénomène semblable a été observé récemment auprès des personnes vivant avec le HIV s'engageant dans des sports (Ferez, Thomas, 2012) et auprès de jeunes sportifs (judo, natation) à propos de leurs problèmes cutanés récurrents (Bellay, Héas, 2011). 